\title{
A Mathematical Model of a Micrometastasis
}

\author{
MALCOLM I. G. BLOOR* and MICHAEL J. WILSON \\ Department of Applied Mathematical Studies, The University of Leeds, Leeds LS2 9JT, UK
}

(Received 10 December 1996)

\begin{abstract}
Experimental evidence indicates that tumour metastases can exist for long periods in a dormant state, with cell proliferation balancing cell death. However, this balance can be upset, by removing the primary tumour for instance, which causes the metastasis to grow, or by administering a substance inhibiting angiogenesis which causes the metastasis to regress. A mathematical model is presented for the growth of a tumour metastasis, which by postulating the possibility of a local imbalance between cell proliferation and cell death through apoptosis, is able to explain some of these observations. A prediction of the model is that at any position within the metastasis there will be a radial movement of cells, even in the dormant state.
\end{abstract}

Keywords: Metastasis, Tumour, Mathematical Modelling

\section{INTRODUCTION}

The in vivo development of cancer, in particular the distant spread of solid tumours, typically starts with the intiation and growth of a primary cancer at some location in the body, followed by the spread to other organs. Metastasis is name of the process whereby malignant cells are 'released' from a primary and spread to other organs of the body where they can lodge and grow to form secondary tumours. In fact, it is tumour metastasis that is the major cause of mortality in cancer patients (Holmgren et al., 1995).

The growth of solid cancers is a complicated phenomenon, but broadly two distinct phases can be distinguished: an initial avascular phase, followed by vascularization (Folkman, 1976, 1985).
During the avascular phase a tumour lacks its own network of blood vessels, and must obtain nutrients (in particular oxygen) and remove waste products by diffusion. If detected in a patient, or when (more commonly) grown in an experimental animal, an avascular tumour is typically less than a few millimetres in diameter, and often appears to be in a steady, dormant state with cell death balancing cell proliferation (Folkman, 1976); it is not unlikely that a few millimetres represents the largest size to which a tumour can grow whilst satisfying its nutrient requirements solely by diffusive transport.

Solid tumours may remain in a dormant, avascular, state for years, before some event triggers the vascular phase of development during which the tumour cells stimulate angiogensis, the process whereby a cancer effects the development of

${ }^{*}$ Corresponding author. 
its own network of blood vessels by growth from neighbouring capillary beds (Folkman, 1976, 1985, 1995). Angiogenesis is initiated by the release from the tumour of substances which produce the growth of new capillaries towards the tumour which eventually becomes penetrated by these vessels (Folkman, 1976, 1985). Very rapid growth of the primary then follows until it is a centimetre or more in size, whereupon its growth often slows down.

Vascularization of the primary is an event of great clinical significance because, although metastasis can sometimes occur before the vascular phase by spread through the lymphatic system, upon vascularization the rate at which malignant cells are released from the primary tumour and spread dramatically increases. Indeed, the number of cells shed by the primary seems to be directly proportional to its mass.

Even though physically separated from its secondaries, the presence of the primary tumour can sometimes have a profound effect on the development of metastases (Folkman, 1995). For instance, for certain cancers the removal of the primary tumour can result in the 'awakening' of occult and distant metastases from a dormant state - in which they may be described, in terms of size, as micrometastases - into a phase of rapid growth (Woodruff, 1980, 1990).

In the absence of angiogensis, metastatic tumour cells may form microscopic perivascular 'cuffs' around a capillary at the location where originally a clonogenic cell left the circulation (Holmgren et al., 1995, Folkman, 1995). In the lungs of a mouse, such cuffs are no larger than about $150 \mu \mathrm{m}$ (Folkman, 1995) or about 10 cells (Holmgren et al., 1995), in radius. A micrometastasis may remain in a dormant avascular state with a high cell proliferation rate balancing a high cell death rate by apoptosis, a form of programmed cell death which differs from necrosis in many ways and is usually characterized by single-cell death in the midst of living cells (Thompson, 1995, Steller, 1995, Bellamy et al., 1995). The onset of angiogenesis can be triggered by the removal of the primary tumour - although Folkman (1995) notes that for certain other mouse tumours micrometastases may not become angiogenic, i.e. the angiogenesis genes are not activated, even 3.5 months after removal of the primary tumour.

O'Reilly et al. (1994) report a mouse model in which a primary inhibits the growth of its remote metastases, but upon the removal of the primary the metastases become vascularized and grow. To explain their observations, O'Reilly et al. (1994) suggest a mechanism whereby the primary initiates its own neovascularization by an excess of angiogenic stimulators in excess of angiogenic inhibitors. However the inhibitors, having a longer half-life in the circulation than the stimulators, reach the vascular bed of the metastases in excess of the stimulators from the primary and any produced by the secondary, and hence prevent the neovascularization and growth of the metastases. The specific inhibitor that O'Reilly et al. identified and studied in their experiments they named angiostatin, which they were able to show mediated, at least in part, the inhibition of metastases by a mouse primary tumour.

Based upon their studies of dormant lung metastases in mice, Holmgren et al. (1995) discuss the balance between cell proliferation and cell death through apoptosis in the presence of angiogenesis suppression. In dormant metastases, they found both a high proliferation index and a high apoptotic index. Correspondingly, microscopic examination of dormant metastases in the lungs of laboratory mice showed no sign of vascularization and no sign of necrosis. The apoptotic indices of quiescent metastases (primary present) were compared to that of exponentially growing metastases (primary removed), and found to be about three times larger in animals where the primary tumour was still present. In addition, Holmgren et al. (1995) also showed that for mice where the primary tumour had been removed, the apoptotic index in lung metastases of animals injected with an angiogenesis inhibitor (TNP-470) was about three times larger than the apoptotic index in lung metastases of animals injected with saline.

Their findings led Holmgren et al. (1995) to conclude that in a dormant state micrometastases are 
able to balance a high cell proliferation rate by a high rate of cell death, elevated by increased apoptosis caused by inhibition of angiogenesis. Their studies showed that angiogenesis significantly lowered apoptosis in metastases, although their high cell proliferation rate remained unaltered.

The effect of angiostatin on a primary tumour is discussed in later work by O'Reilly et al. (1996) who investigated its effects on three human and three murine primary tumours implanted in a mouse model. They describe experiments in which the administration of angiostatin caused the human primaries to regress to a microscopic dormant state; the growth of the murine tumours was also inhibited by angiostatin but did not reach a dormant state (because, O'Reilly et al. (1996) assumed, the murine turnours had been selected, over the years, on the basis of their angiogeneticity). O'Reilly et al. (1996) measured the proliferative and apoptotic indices of the tumour cells and showed that although the proliferative index was the same in both control and angiostatin-treated animals, the apoptotic index was significantly higher in the latter group.

The mechanism whereby angiostatin increases the apoptotic index is unknown, but O'Reilly et al. (1996) mention various suggestions, e.g. the inhibition of capillary growth deprives the tumour cells of necessary factors provided by the endothelium. However, on the basis of this work, they suggest a new paradigm for anticancer treatment, 'dormancy therapy', based upon the administration of antiangiogenesis agents such as angiostatin.

We present here a simple mathematical model for the development of a micrometastasis. We assume a cylindrically symmetric geometry as a convenient model for a perivascular cuff. A local imbalance in the rates of apoptosis and proliferation near to the capillary causes the micrometastasis to grow, although the micrometastasis eventually reaches a dormant state for reasons described in the next section. We assume that angiogenesis is being suppressed by a factor such as angiostatin, and, furthermore, that the presence of this factor affects the rate of apoptosis.

\section{A MATHEMATICAL MODEL FOR A MICROMETASTASIS}

Most mathematical models of the growth of solid tumours have concentrated upon the development of the primary tumour, particularly in the avascular phase of growth (Adam, 1991). Various models of an avascular primary tumour have been put forward since the mid-1960s (Burton, 1966; Deakin, 1975; Greenspan, 1972, 1976; McElwain and Ponzo, 1977; Chaplain and Sleeman, 1993; Sleeman, 1996), all of which account, at least in part, for the development of the tumour and the changes in its structure, by considering the effects of nutrient (usually oxygen) diffusion and consumption on the proliferation and death (usually through necrosis) of tumour cells.

The models referenced above adopt a continuum approach in that the properties of the tumour cells are modelled in an aggregative sense or continuum, not at the level of individual cells. Here we adopt a similar approach to account for the growth of a micrometastasis. The specific geometry we consider is a perivascular cuff growing around a capillary vessel, and for simplicity we assume cylindrical symmetry in our model. We assume that the cancer cells obtain nutrient, in this case oxygen, by diffusion from the capillary which they surround. As noted above, experimentally, micrometastases are observed to be around 10 cells in radius and so some questions may be asked about the validity of a continuum model. However, the discrete nature of the problem does not alter the underlying physical processes, and thus a continuum model will capture perfectly adequately the overall structure and growth of the cuff without any loss of physical insight.

Like other mathematical models for tumour growth, to account for the existence of a finite equilibrium radius for a tumour, a mechanism for the loss of cell volume is assumed, but whereas most other models assume that volume loss occurs through necrosis, the present model assumes volume loss through apoptosis. Apoptosis is a form of programmed cell death that is widely accepted as being of crucial importance to the normal development and homeostasis of 
multicellular organisms (Steller, 1995). In contrast to necrosis, which is a pathological form of cell death due to injury resulting in rapid cell swelling and lysis, apoptosis results in cell shrinkage and volume loss (Thompson, 1995, Bellamy et al., 1995). Apoptosis has a mechanism for the loss of cell volume has been considered in the context of spherical tumours by McElwain and Morris (1978) and Byrne and Chaplain (1995a, 1996), who found that it was possible to obtain a dormant state even in the absence of a non-necrotic core.

It has only relatively recently become appreciated that the regulation of cell death is just as complex as the regulation of cell production, and many diseases can be characterized as either disorders of too large a death rate, such as AIDS, whereas others can be characterized as being disorders of too small a death rate, such as cancer (Thompson, 1995). Apoptosis appears to be triggered by a variety of extrinsic and intrinsic signals, and Thompson (1995) reports that most cells in the body are programmed to commit suicide if they do not receive appropriate chemical signals from their environment. This may explain the observations described above (O'Reilly, 1994, 1996; Folkman, 1995; Holmgren et al., 1995) that treatment with angiostatin, which inhibits angiogensis and hence presumably reduces the accessibility to the tumour cells of various necessary factors, carried by the blood or provided by the endothelium for instance, raises the apoptotic index in metastases. In any event, the model ${ }^{p}$ described below can take into account the possibility that the apoptotic index in the metastasis is affected by the angiostatin concentration in the central capillary.

The present model differs from earlier work in that it considers the growth of a mass of cancer cells where the source of nutrient is at the centre of the mass, i.e. the capilliary around which the micrometastasis is growing. It also makes explicit a fact that has hitherto only been implicit in certain tumour models, namely that there must be a radial movement of cells as the tumour grows.

We consider a cylindrically symmetric cuff of cancer cells around a central capillary, which we shall take to be of radius $r_{\mathrm{m}}$. Suppose that at a given moment the outer radius of the cuff is $R(t)$. At any point in the microscopic tumour, a distance $r$ from the centre of the capillary, there will be a local volume proliferation rate $k$ (cell volume created/unit volume/unit time; units $\mathrm{T}^{-1}$ ) and a local apoptotic volume death rate $\alpha$ (cell volume destroyed/unit volume/unit time; units $\mathrm{T}^{-1}$ ). Any local imbalance in proliferation and apoptosis will produce a nonzero cell velocity $\underline{u}$ at that point, where

$$
\nabla \cdot \underline{u}=(k-\alpha)
$$

Since we are assuming cylindrical symmetry in this paper we will use a cylindrical polar coordinate system, so that $\underline{u}=u \underline{e}_{r}$, where $e_{r}$ is a unit vector in the radial direction, and so equation (1) may be written

$$
\frac{\partial(r u)}{\partial r}=r(k-\alpha)
$$

where $u$ is the radial velocity of the cellular material, where we have assumed that after apoptotic death a cell ceases to occupy any volume. Below, we solve equation (2) subject to boundary conditions (see equation (9)), in order to obtain the radius of the micrometastasis as a function of time.

The diffusion of nutrient (which we will take to be oxygen) out from the central capillary we will assume takes place on a much shorter time-scale than the typical time-scales for cell proliferation and death (a natural assumption to make, see for example Adam and Noren (1993), so that we have a quasi-steady model in which the oxygen concentration $c$ obeys the equation

$$
\frac{D}{r} \frac{\partial}{\partial r}\left(r \frac{\partial c}{\partial r}\right)=s
$$

where $s=$ nutrient consumption rate and $D=$ nutrient diffusion coefficient, both assumed to be constant while the cells of the micrometastasis are viable. A further condition necessary for the quasisteady assumption is that the time-scale for diffusion is much less than a typical 'dynamical' time-scale $R / U$, where $U$ is a typical cell velocity.

For the micrometastases being considered, there is no evidence that the cancer cells are becoming less viable due to nutrient starvation. However, some 
authors, in somewhat different circumstances, have considered a nutrient consumption rate which is a function of nutrient concentration, in particular a consumption rate which is constant until the concentration drops to a certain level, and then falls, often linearly, to zero as the concentration drops still further and the cells cease to be viable; see for example McElwain and Ponzo (1977) or Byrne and Chaplain (1995a). Although this assumption could be built into the model, the qualitative aspects of the results would be unchanged but at the cost of a more complicated mathematical treatment.

Assuming that the oxygen concentration at the capillary wall is $c_{0}$ and the nutrient flux $\left(-D r_{\mathrm{m}}(\partial c / \partial r)\right)$ is $m$, the solution to equation (3) which satisfies these boundary conditions can be written

$$
c=c_{0}-\frac{1}{D}\left(m+\frac{s r_{\mathrm{m}}^{2}}{2}\right) \ln \left(r / r_{\mathrm{m}}\right)+\frac{s}{4 D}\left(r^{2}-r_{\mathrm{m}}^{2}\right)
$$

Since Holmgren et al. (1995) report no sign of necrosis in their observations of micrometastases, we will assume that the concentration $c$ experienced by the micrometastasis never gets so low that necrosis occurs, and hence that its radius remains much smaller than $(2 \mathrm{~m} / \mathrm{s})^{\frac{1}{2}}$, which is the radius at which $r(\partial c / \partial r)=0$ (assuming $r_{\mathrm{m}}$ to be small), i.e. the radius, $r_{\mathrm{e}}$, the micrometastasis would need to be if it were to consume all the nutrient flux from the central capilliary.

Putting $r_{\mathrm{e}}^{2} \approx(2 \mathrm{~m} / \mathrm{s})$, equation (4) can be written

$$
c=c_{0}-\frac{s}{2 D}\left(r_{\mathrm{e}}^{2}+r_{\mathrm{m}}^{2}\right) \ln \left(r / r_{\mathrm{m}}\right)+\frac{s}{4 D}\left(r^{2}-r_{\mathrm{m}}^{2}\right)
$$

and, if we assume that throughout its growth the radius $r$ of the micrometastasis remains much smaller than $r_{\mathrm{e}}$ i.e. $r_{\mathrm{m}}<r \ll r_{\mathrm{e}}$, the oxygen concentration the micrometastasis experiences can be approximated by

$$
c=c_{0}-\frac{s r_{\mathrm{e}}^{2}}{2 D} \ln \left(r / r_{\mathrm{m}}\right)
$$

since the third term of equation (5) remains smaller than the second for $r \ll r_{\mathrm{e}}$. In other words the amount of nutrient consumed by the cells is a small fraction of the total amount available, i.e. there is no significant variation in the oxygen concentration due to consumption.

In common with other models, see for example McElwain and Ponzo (1977), McElwain and Morris (1978), Byrne and Chaplain (1995a, 1996), it is assumed here that the cell proliferation rate is a function of nutrient concentration which for simplicity we take to be of the form $k \propto$ (constant + constant $c$ ), which, given equation (6), implies that

$$
k=k_{0}-k_{2} \ln \left(r / r_{\mathrm{m}}\right)
$$

with $k_{0}, k_{2}>0$.

The rate of apoptosis $\alpha$ we initially take to be a constant $\alpha_{0}$ throughout the micrometastasis at a given moment. We (implicitly) assume a functional relation between the rate of apoptosis and the concentration of the angiogenesis inhibitor in the central capillary, in that in the discussion below we consider the effect of a time-varying $\alpha$ as a presumed consequence of a change in the inhibitor concentration. However, given the uncertainties surrounding the mechanism whereby angiostatin affects the rate of apoptosis in a tumour, we do not assume an explicit functional form for the relationship between the rate of apoptosis and inhibitor concentration, although the model could, nevertheless, be modified to take into account effects such as diffusion and depletion due to absorption of the inhibitor. Note that for the micrometastasis to start to grow, we require $k_{0}>\alpha_{0}$.

Integrating equation (2) from the edge of the capillary to the current outer radius $R(t)$ of the micrometastasis gives

$$
[r u]_{r_{\mathrm{m}}}^{R}=\int_{r_{\mathrm{m}}}^{R} r(k-\alpha) \mathrm{d} r
$$

and using equation (7) and imposing the boundary condition that $u=0$ at $r=r_{\mathrm{m}}$, i.e. the micrometastasis remains in contact with the capillary wall and there is no flux of cancer cells across the wall, we obtain

$$
R \frac{\mathrm{d} R}{\mathrm{~d} t}=\int_{r_{\mathrm{m}}}^{R} r\left(k_{0}-k_{2} \ln \left(r / r_{\mathrm{m}}\right)-\alpha_{0}\right) \mathrm{d} r
$$


where the cell velocity at the outer edge of the micrometastasis is the rate at which the micrometastasis expands into the surrounding medium, i.e. $\left.u\right|_{r=R(t)}=(\mathrm{d} R / \mathrm{d} t)$.

The right-hand side of equation (9) can be evaluated to give

$$
\begin{aligned}
R \frac{\mathrm{d} R}{\mathrm{~d} t}= & \frac{1}{2}\left(R^{2}-r_{\mathrm{m}}^{2}\right)\left(k_{0}+\frac{k_{2}}{2}-\alpha_{0}\right)-\frac{k_{2}}{2} R^{2} \\
& \ln \left(R / r_{\mathrm{m}}\right)
\end{aligned}
$$

It is useful in what follows to change to a nondimensional time variable defined thus,

$$
\tau=k_{0} t
$$

so that the time-scale for the evolution of the micrometastasis is clearly related to a measure of the cell-cycle time. We can then rewrite equation (10) to obtain

$$
R \frac{\mathrm{d} R}{\mathrm{~d} \tau}=\frac{1}{2}\left(R^{2}-r_{\mathrm{m}}^{2}\right)\left(1+\frac{\gamma}{2}-\eta\right)-\frac{\gamma}{2} R^{2} \ln \left(R / r_{\mathrm{m}}\right)
$$

where we have defined the dimensionless parameters $\eta$ and $\gamma$ - which characterize the relative importance of apoptosis to proliferation at the capillary and the radius at which the proliferation index falls to zero $\left(r_{\mathrm{m}} \exp (1 / \gamma)\right)-$ thus

$$
\eta=\frac{\alpha}{k_{0}} \quad \gamma=\frac{k_{2}}{k_{0}}
$$

As noted above, for the micrometastasis to start to grow at all, we require $\eta<1$.

From equation (12) it can be seen that the micrometastasis stops growing in size, i.e. $(\mathrm{d} R / \mathrm{d} t)=0$, at a radius $R_{\mathrm{d}}$ given by

$$
R_{\mathrm{d}}=r_{\mathrm{m}} \exp \left(\frac{1+\frac{\gamma}{2}-\eta}{\gamma}\right)
$$

if $R_{\mathrm{d}}^{2} \gg r_{\mathrm{m}}^{2}$ (Holmgren et al. (1995) observe that $\left.R_{\mathrm{d}} \approx 10 r_{\mathrm{m}}\right)$. $R_{\mathrm{d}}$ represents the maximum radius to which the micrometastasis can grow given the previaling conditions, and, presumably, corresponds to its dormant state.

Also, $\mathrm{d} R / \mathrm{d} t=0$ when $R=r_{\mathrm{m}}$, and so to avoid an obvious inconsistency in the model, i.e. if starting precisely at $r=r_{\mathrm{m}}$ the micrometastasis cannot grow, we assume that the actual starting radius for the micrometastasis is $R_{0}=r_{\mathrm{m}}+(1 \mathrm{cell}$ layer thickness).

A prediction of the model is that, even in a dormant state, at any radius there will be a radial migration of cancer cells, where the cell velocity as a function of radius

$$
u(r)=\frac{1}{2 r}\left(1+\frac{\gamma}{2}-\eta\right)\left(r^{2}-r_{\mathrm{m}}^{2}\right)-\frac{\gamma}{2} r \ln \left(r / r_{\mathrm{m}}\right)
$$

is obtained from equation (8) (cf. McElwain and Pettet (1993) who consider a model of a multicellular spheroid in which there is radial migration of cells towards the centre).

As a check on the internal consistency of the model we can consider the magnitude $U$ of the typical sort of velocities involved. For convenience we take the maximum velocity which, from equation (15), can be shown to be $\frac{1}{2} \gamma \exp \{(1-$ $\gamma / 2-\eta) / \gamma$. Comparing the value of $R_{\mathrm{d}} / U(=$ $\left.(2 / \gamma) \mathrm{e}^{1}\right)$ with the cell doubling time $\ln 2$ (in nondimensional units), we find that we require the condition $7.8 \gg \gamma$.

To obtain the approximate time development of the cuff, by making the assumption that for most of its growth $r \gg r_{m}$ equation (12) can be integrated to give

$$
R=r_{\mathrm{m}}\left(\frac{R_{0}}{r_{\mathrm{m}}}\right)^{\mathrm{e}^{-\frac{\gamma \tau}{2}}} \exp \left\{\frac{\left(1+\frac{\gamma}{2}-\eta\right)}{\gamma}\left(1-\mathrm{e}^{-\frac{\gamma \tau}{2}}\right)\right\}
$$

where the initial condition $R(0)=R_{0}$, defined as above, has been imposed. Notice that $R \rightarrow R_{\mathrm{d}}$ as $t \rightarrow \infty$. For a more accurate description of the growth, equation (12) can of course be integrated.

In order to obtain results consistent with the assumptions of the model, certain constraints must be placed upon the various parameters controlling the growth. For instance, the dormancy radius $R_{\mathrm{d}}$ should be less than the radius at which cell proliferation drops to zero $\left(r_{\mathrm{m}} \exp (1 / \gamma)\right)$, which requires that $\eta>\gamma / 2$. Furthermore, in order for the assumption underlying the derivation of equation (14) to be valid, i.e. $R_{\mathrm{d}}^{2} \gg r_{\mathrm{m}}^{2}$, we require $\exp ((1+\gamma / 2-$ $\eta) / \gamma) \gg 1$. 
Figures 1 and 2 illustrate the effects of varying the relative importance of apoptosis and proliferation (quantified by the value of $\eta$ ), and the effect of changing the radius at which cell proliferation stops (determined by the value of $\gamma$ ). The solutions have been obtained by integrating equation (12) numerically.

Figure 1 shows that for fixed $\gamma$, as the value of $\eta$ increases, i.e. as apoptosis becomes more important, then, as indicated by equation (14), the dormancy radius decreases. Note also that the value of $\eta$ has little effect on the time taken to reach the dormancy radius, which again is consistent with equation (16).
Figure 2 shows that for a fixed $\eta$, as the radius at which proliferation stops is decreased, ie. as the value of $\gamma$ increases, so the dormancy radius is decreased and the time taken to get within a fixed fraction of this radius decreases - behaviour consistent with equation (16).

Note that since Holmgren et al. (1995) observe no signs of necrosis, we have not included the effects of necrosis in the model and have assumed that the dormancy radius for the micrometastasis is less than the radius at which the nutrient concentration goes to zero (although necrosis may well begin at low, but nevertheless non-zero, nutrient concentrations).

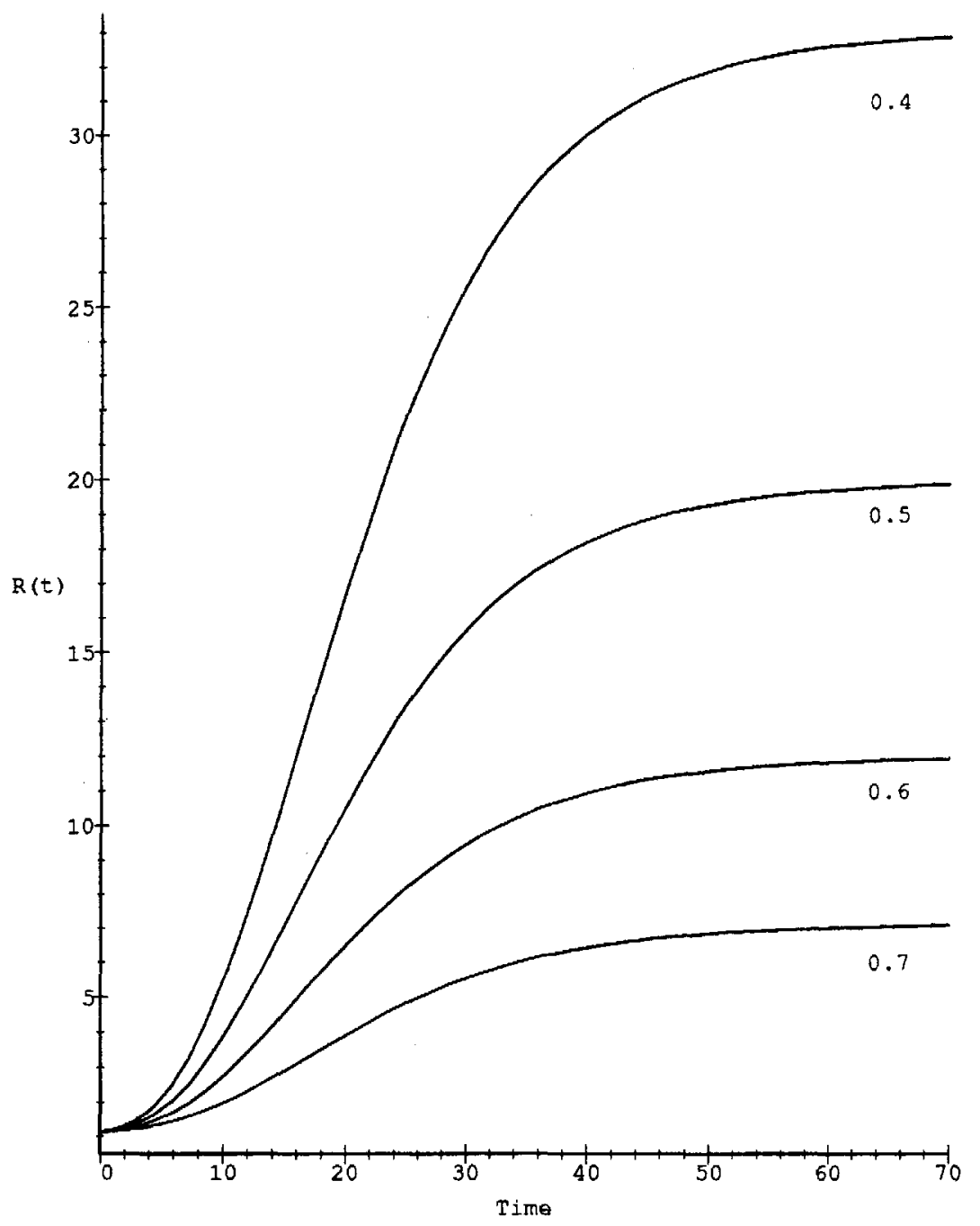

FIGURE 1 The effect of $\eta$ upon growth of micrometastasis. In all plots $r_{\mathrm{m}}=1, \gamma=0.2, R_{0}=1.1$. 


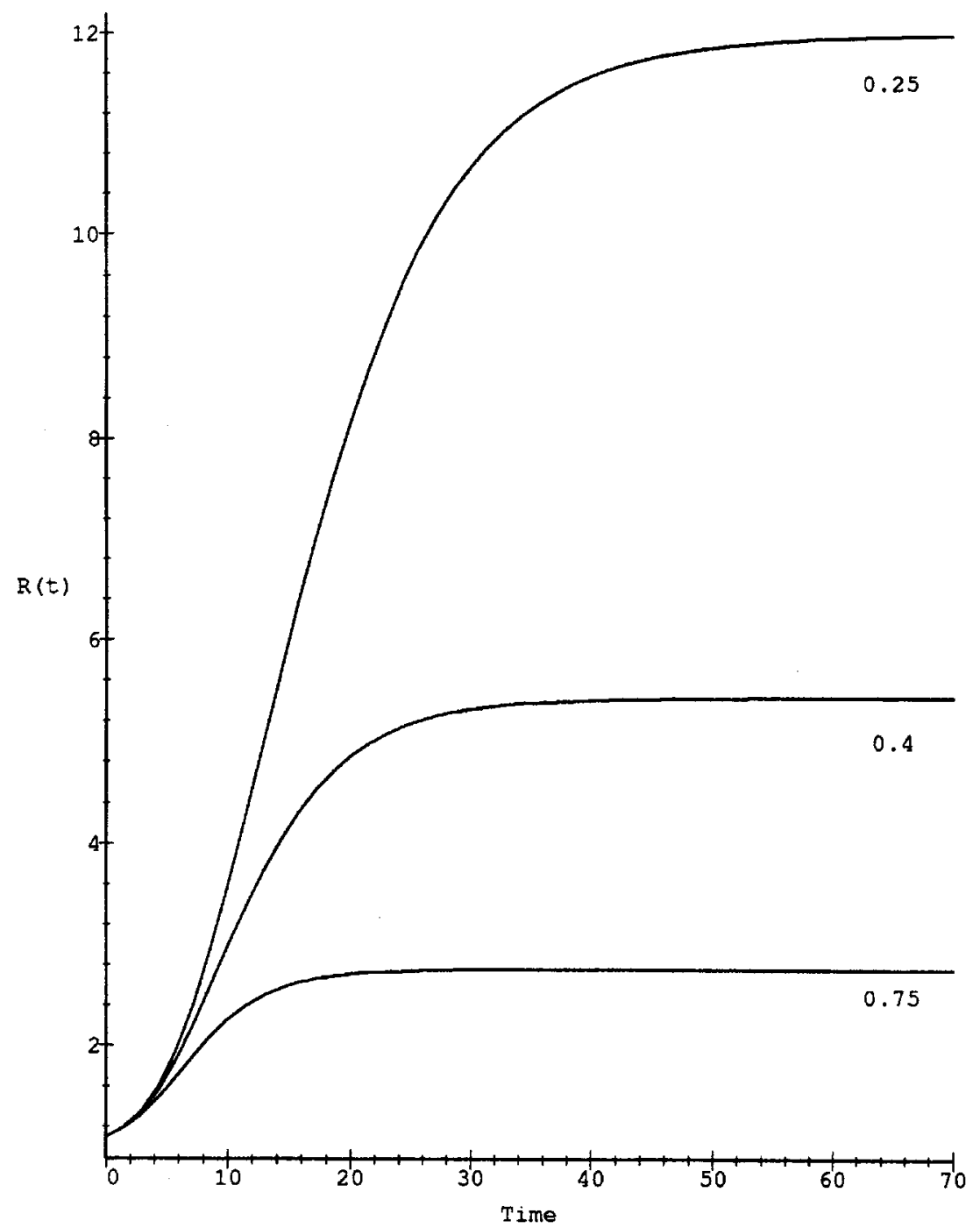

FIGURE 2 The effect of $\gamma$ upon growth of micrometastasis. In all plots $r_{r m m}=1, \eta=0.5, R_{0}=1.1$.

THE EFFECT OF A DIFFERENT FUNCTIONAL FORM FOR THE PROLIFERATION RATE

In this section we assess the effect of having a different functional form for the variation of proliferation rate with nutrient concentration. For the sake of example we assume that the proliferation rate remains constant at $k_{0}$ for values of the nutrient concentration $c$ above a critical value $\hat{c}$, and then drops to zero for values of $c$ below $\hat{c}$, the cancer cells becoming dormant rather than $c$ becoming so low that necrosis begins, i.e.

$$
k= \begin{cases}k_{0} & \text { if } c>\hat{c} \\ 0 & \text { if } c<\hat{c}\end{cases}
$$

If we assume that the nutrient concentration varies as a function of radius as described by equation (6), the critical radius $\hat{r}$ at which the nutrient concentration reaches $\hat{c}$ is given by

$$
\hat{r}=r_{\mathrm{m}} \exp \left(\frac{2 D\left(c_{0}-\hat{c}\right)}{s r_{\mathrm{e}}^{2}}\right)
$$


The time evolution of the outer radius $R$ of the micrometastasis can be obtained by integrating the right-hand side of equation (8).

For $r<\hat{r}$ we get

$$
R \frac{\mathrm{d} R}{\mathrm{~d} \tau}=\frac{1}{2}\left(R^{2}-r_{\mathrm{m}}^{2}\right)(1-\eta)
$$

which may be integrated to give

$$
R^{2}=r_{\mathrm{m}}^{2}+\left(R_{0}^{2}-r_{\mathrm{m}}^{2}\right) \exp ((1-\eta) \tau)
$$

where $R(0)=R_{0}$.
When the micrometastasis has grown so that $R>\hat{r}$, the right-hand side of equation (8) may be integrated to give

$$
R \frac{\mathrm{d} R}{\mathrm{~d} \tau}=\frac{1}{2}\left(\hat{r}^{2}-r_{\mathrm{m}}^{2}\right)+\frac{1}{2}\left(r_{\mathrm{m}}^{2}-R^{2}\right) \eta
$$

which may be solved with the initial condition $R(\hat{\tau})=\hat{r}$ to give

$$
\begin{aligned}
R^{2}= & \frac{1}{\eta}\left\{\left(\hat{r}^{2}-(1-\eta) r_{\mathrm{m}}^{2}\right)-\left(\hat{r}^{2}-r_{\mathrm{m}}^{2}\right)(1-\eta)\right. \\
& \exp (-\eta(\tau-\hat{\tau}))\}
\end{aligned}
$$

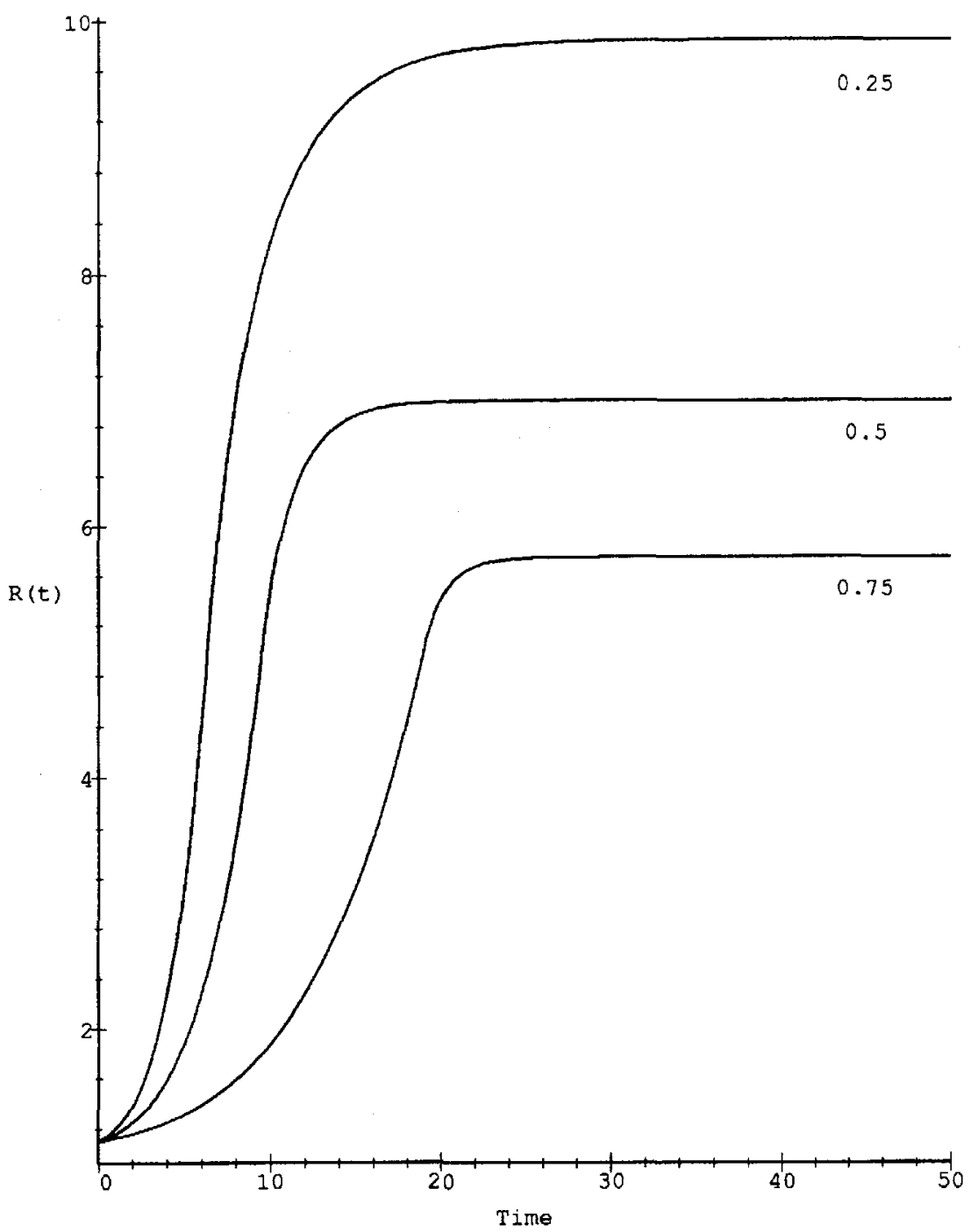

FIGURE 3 The effect of $\eta$ upon growth of micrometastasis. In all plots $r_{\mathrm{m}}=1, \hat{r}=5, R_{0}=1.1$. 
where $\hat{\tau}$, the time taken for the micrometastasis to grow to $\hat{r}$, is easily found from equation (20) to be

$$
\hat{\tau}=\frac{1}{(1-\eta)} \ln \left(\frac{r^{2}-r_{\mathrm{m}}^{2}}{R_{0}^{2}-r_{\mathrm{m}}^{2}}\right)
$$

Note that equation (22) shows that as $\tau \rightarrow \infty, R^{2} \rightarrow$ $r_{\mathrm{m}}^{2}+\left(\hat{r}^{2}-r_{\mathrm{m}}^{2}\right)(1 / \eta)$, the dormancy radius.

Like Figures 1 and 2 for the previous model, Figures 3 and 4 illustrate the effects of varying the relative importance of apoptosis and proliferation (quantified by the value of $\eta$ ), and the effect of changing the radius at which cell proliferation stops (determined by the value of $\hat{r}$, for the modified model presented in this section).

Figure 3 shows that for constant $\hat{r}$ increasing the relative importance of apoptosis, i.e. increasing $\eta$ has the effect of decreasing the dormancy radius and also increasing the time it takes the micrometastasis to get to within a fixed fraction of this radius.

Figure 4 shows that for constant $\eta$ the effect of decreasing the critical radius at which proliferation stops, i.e. decreasing the value of $\hat{r}$, has the effect of decreasing the eventual dormancy radius.

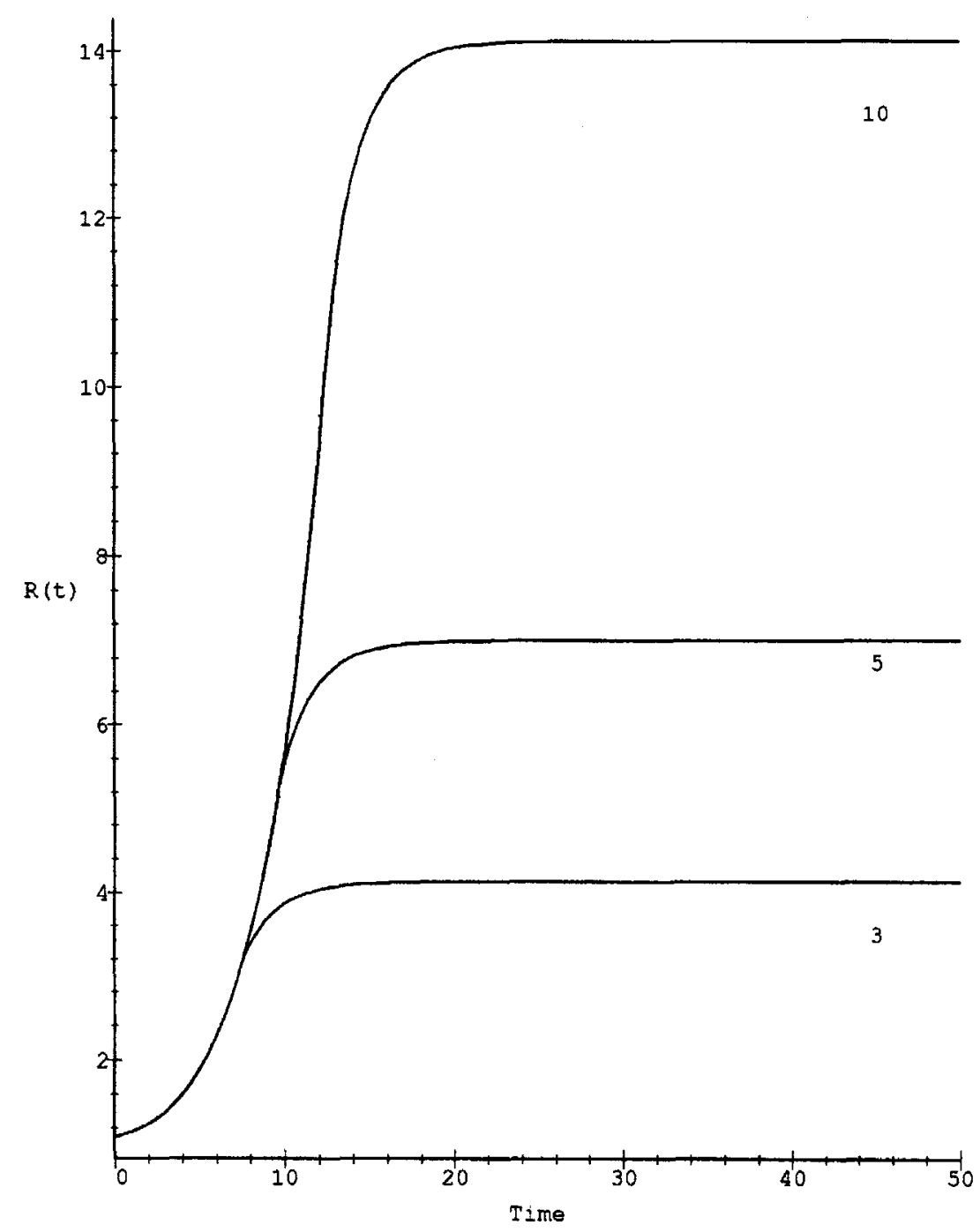

FIGURE 4 The effect of $\hat{r}$ upon growth of micrometastasis. In all plots $r_{\mathrm{m}}=1, \eta=0.5, R_{0}=1.1$. 
The results presented in this section indicates that changing the functional form of the dependence of proliferation rate on nutrient concentration may produce quantitative differences in the growth of the micrometastasis, but the qualitative behaviour still remains the same, i.e. growth stops at some finite radius. The crucial point being that the proliferation rate decreases with nutrient concentration which in turn decreases with radius, causing a local excess of cell death over cell proliferation in the outer regions of the micrometastasis.

\section{THE EFFECT OF VARYING THE APOPTOTIC INDEX}

There is some evidence that the size of the primary tumour may affect the inhibition of the metastases (O'Reilly, et al. 1994). In this section we consider the effect of an increase in the rate of apoptosis, due perhaps to a change in the angiostatin concentration.

There is also evidence that angiostatin has a significant half-life in the body. For example, a figure of 5 days is quoted by Folkman (1995) for the time taken for angiostatin to disappear from the human circulation, while a half-life of 4-6 hours is quoted by O'Reilly et al. (1996) for human angiostatin in mice. So it is perhaps unrealistic to consider instantaneous changes in $\alpha$, although, if we take the cell doubling time to be about 48 hours, the time-scales just quoted are short in comparison to the growth time-scale in the above model. However, as a model for the variation in the apoptosis rate, one might consider a gradual variation. For example, suppose that for $\tau \leq \tau_{0}$, the non-dimensional rate of apoptosis changes according to the equation

$$
\eta=\eta_{0}-\frac{1}{2}\left(\eta_{0}-\eta_{1}\right)\left(1+\tanh \left(\left(\tau-\tau_{0}\right) / T\right)\right.
$$

where $0<\eta_{0}<\eta_{1}$, so that $\eta$ increases to $\eta_{1}$ over a characteristic (non-dimensional) time-scale $T$.

We can obtain the subsequent time development of the micrometastasis by integrating equation (12), with $R_{\mathrm{d}}$ from equation (14) as the starting radius, assuming that prior to $\tau_{0}$ the micrometastasis had more or less reached the dormancy radius $R_{\mathrm{d}}$ corresponding to $\eta_{0}$. However before considering this, we note that to obtain the long-time behaviour of the solution we can consider an instantaneous increase in the rate of apoptosis from $\eta_{0}$ to $\eta_{1}$. Similar arguments to those used above show that the time development of the micrometastasis will be governed by the equation

$R \frac{\mathrm{d} R}{\mathrm{~d} \tau}=\frac{1}{2}\left(R^{2}-r_{\mathrm{m}}^{2}\right)\left(1+\frac{\gamma}{2}-\eta_{1}\right)-\frac{\gamma}{2} R^{2} \ln \left(R / r_{\mathrm{m}}\right)$

which gives rise to a new dormancy radius

$$
\bar{R}_{\mathrm{d}}=r_{\mathrm{m}} \exp \left(\frac{1+\frac{\gamma}{2}-\eta_{1}}{\gamma}\right)
$$

Equation (25) may be integrated to give the time development of the cuff

$$
\begin{aligned}
R= & r_{\mathrm{m}}\left(\frac{R_{\mathrm{d}}}{r_{\mathrm{m}}}\right)^{\mathrm{e}-\frac{\gamma\left(\tau-\tau_{0}\right)}{2}} \exp \left\{\frac{\left(1+\frac{\gamma}{2}-\eta_{1}\right)}{\gamma}\right. \\
& \left.\left(1-\mathrm{e}^{-\frac{\gamma\left(\tau-\tau_{0}\right)}{2}}\right)\right\}
\end{aligned}
$$

where the initial condition $R\left(\tau_{0}\right)=R_{d}$ has been used. Note that the radius of the micrometastasis increases towards its new equilibrium radius $\bar{R}_{d}$ over a finite time-scale even though the increase in $\eta$ is instantaneous.

Figure 5 shows the results of some calculations involving the numerical integration of equation (25) where the value of $\eta$ varies with time according to equation (24). At time $\tau_{0}=0$, the value of $\eta_{0}$ is 0.5 and micrometastasis radius is the corresponding dormancy radius (20.08). The three curves in Figure 5 correspond to different values of $\eta(0.6,0.7,0.8)$ (all have the same value of the other parameters) and the time constant $T=20$. As expected, in all three cases the micrometastasis radius decreases with time towards the dormancy radius corresponding to the value of $\eta_{1}$.

Figure 6 shows the effect of changing the timescale $T$ for the apoptosis change. In the five curves 


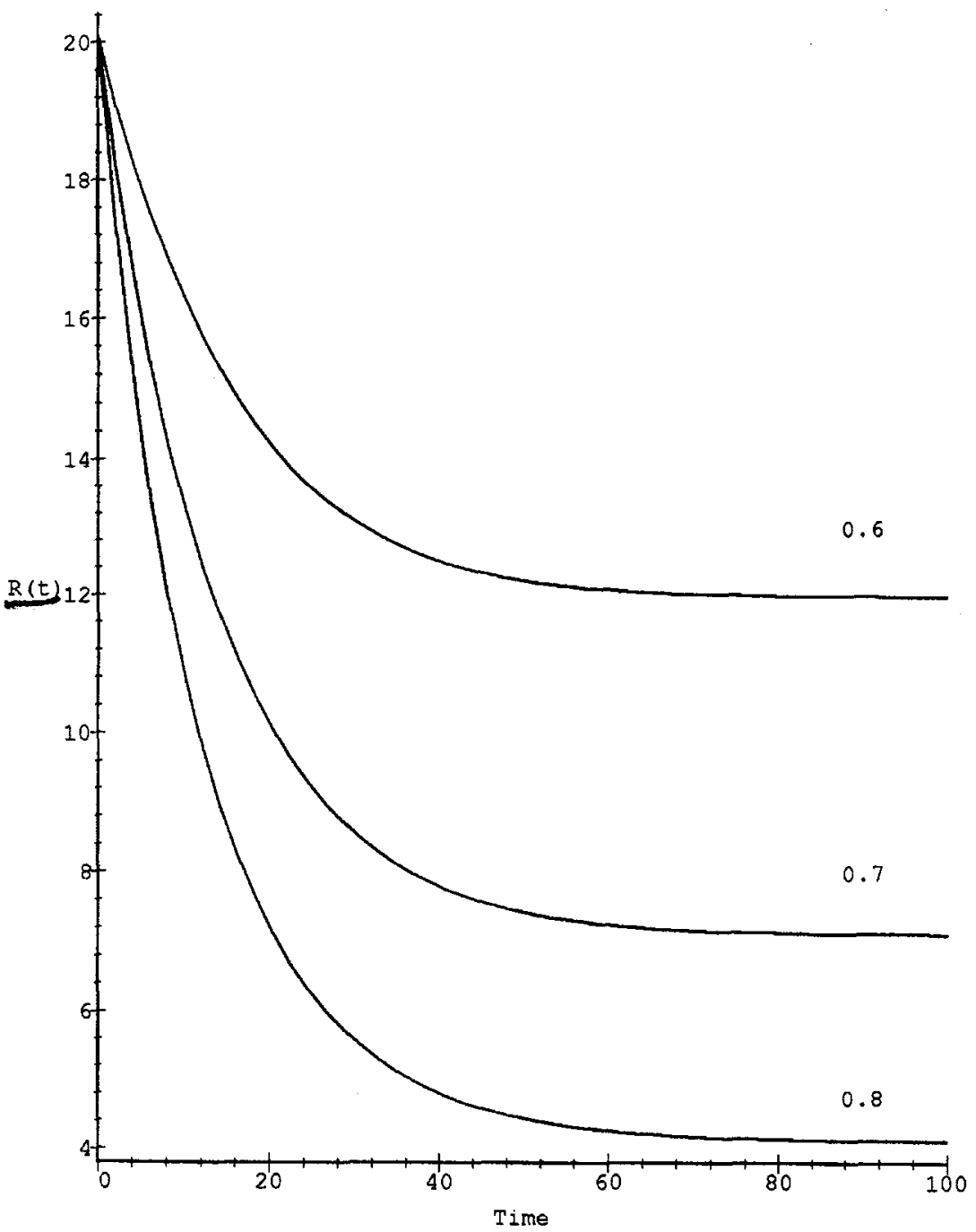

FIGURE 5 The effect of a time-varying apoptosis rate; the number associated with each curve gives the final value of $\eta_{1}$. In all plots $r_{\mathrm{m}}=1, \eta_{0}=0.5, R_{0}=20.08, \gamma=0.2, T=20$.

plotted, $\eta_{0}=0.5, \eta_{1}=0.7$ and $\gamma=0.2$, while the initial radius for the micrometastasis is the dormancy radius corresponding to $\eta_{0}=0.5$. The values of $T$ used are $1,5,10,40$ Note that for large values of $T$ the variation in micrometastasis radius is essentially governed by the time-scale of the variation described by equation (24), whereas if $T$ is sufficiently small the variation in micrometastasis radius is essentially described by equation (27).

In considering the consequences of increasing $\eta$, we are in effect modelling the effects of a dormancy therapy (O'Reilly, et al. 1996) which acts by increasing the rate of apoptosis. From equation (25) we can seen that if we wish to regress the micrometastasis so that $\bar{R}_{\mathrm{d}} \approx r_{\mathrm{m}}$, ie. the micrometastasis is effectively 'removed', we require a value for $\eta_{1}$ of $(1+\gamma / 2)$. Figure 7 shows the results of just such a calculation, where a value of 1.1 has been used for $\eta_{1}$, which, with a value of 0.2 for $\gamma$, should cause the micrometastasis radius to decrease to the value of $r_{\mathrm{m}}$, i.e. 1.0. A value of 1 has been used for $T$ so that the change in micrometastasis radius is close to the fastest possible. Despite this, however, although the micrometastasis radius 


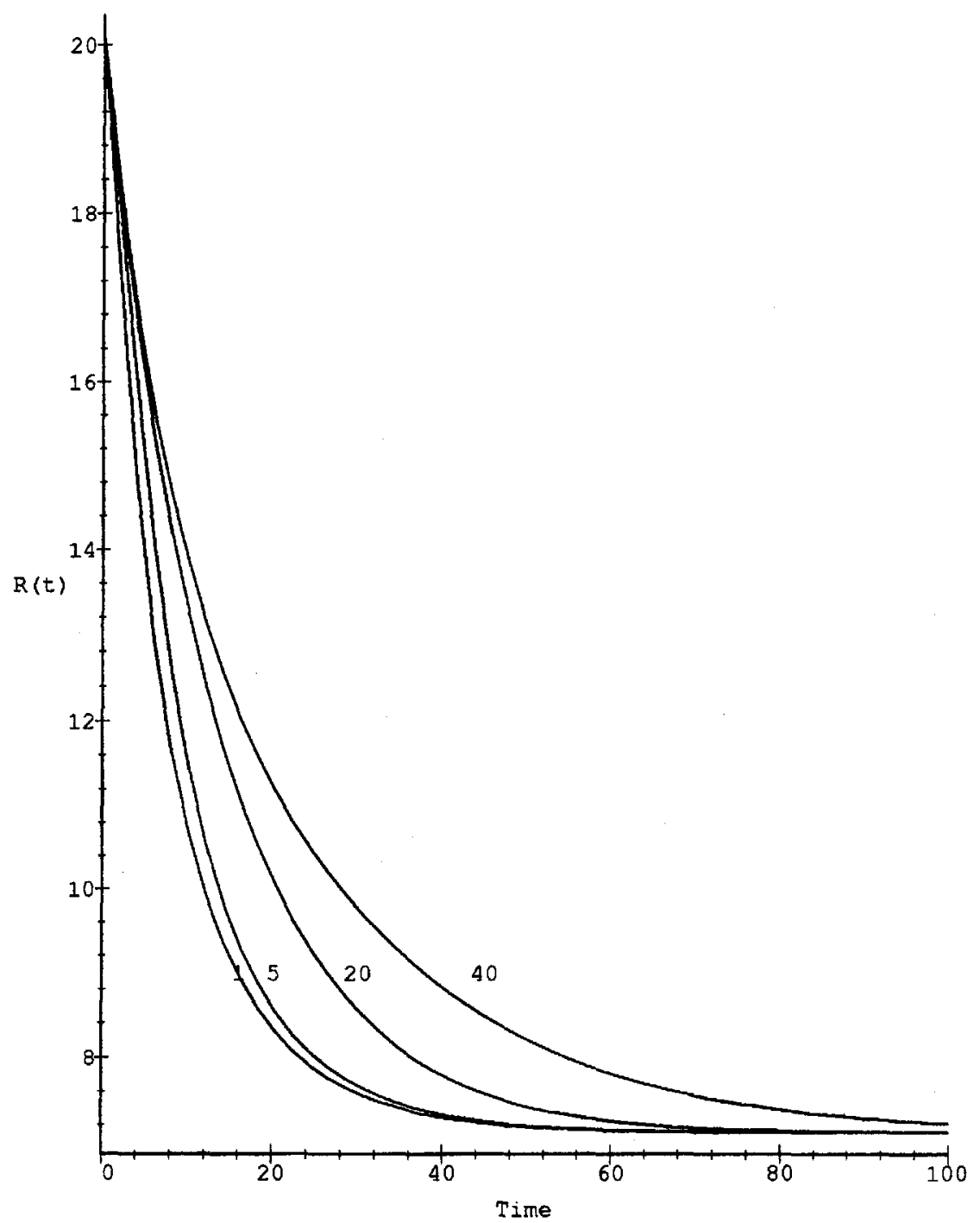

FIGURE 6 The effect of the time-scale for the change in apoptosis rate; the number associated with each curve gives the value of $T$. In all plots $r_{m}=1, \eta_{0}=0.5, \eta_{1}=0.7, R_{0}=20.08, \gamma=0.2$.

does approach the desired value, the time-scale over which it does so is rather longer than $T$, which is, perhaps, inconvenient from the point of view of therapy.

We have not allowed for any variation in the apoptotic index with radius. It has been suggested that cells die through apoptosis more readily if they are deprived of various chemical signals. Thus, it is possible that diffusing out from the central capillary is some factor whose concentration determines the local rate of apoptosis $\alpha$ (in the sense of lowering concentration increases $\alpha$ ), in which case $\alpha$ should vary with radius, and not be treated as a constant in equation (8). However, in the model the effects of varying $\alpha$ are largely interchangeable with the effects of varying the proliferation rate $k$, e.g. $k$ decreasing with increasing $r$, acts, qualitatively, like an $\alpha$ which increases with $r$. Thus although the model can be straight forwardly modified to take into account a radial variation in $\alpha$, the qualitative behaviour of such a case should, in essence, be covered by the above discussion. 


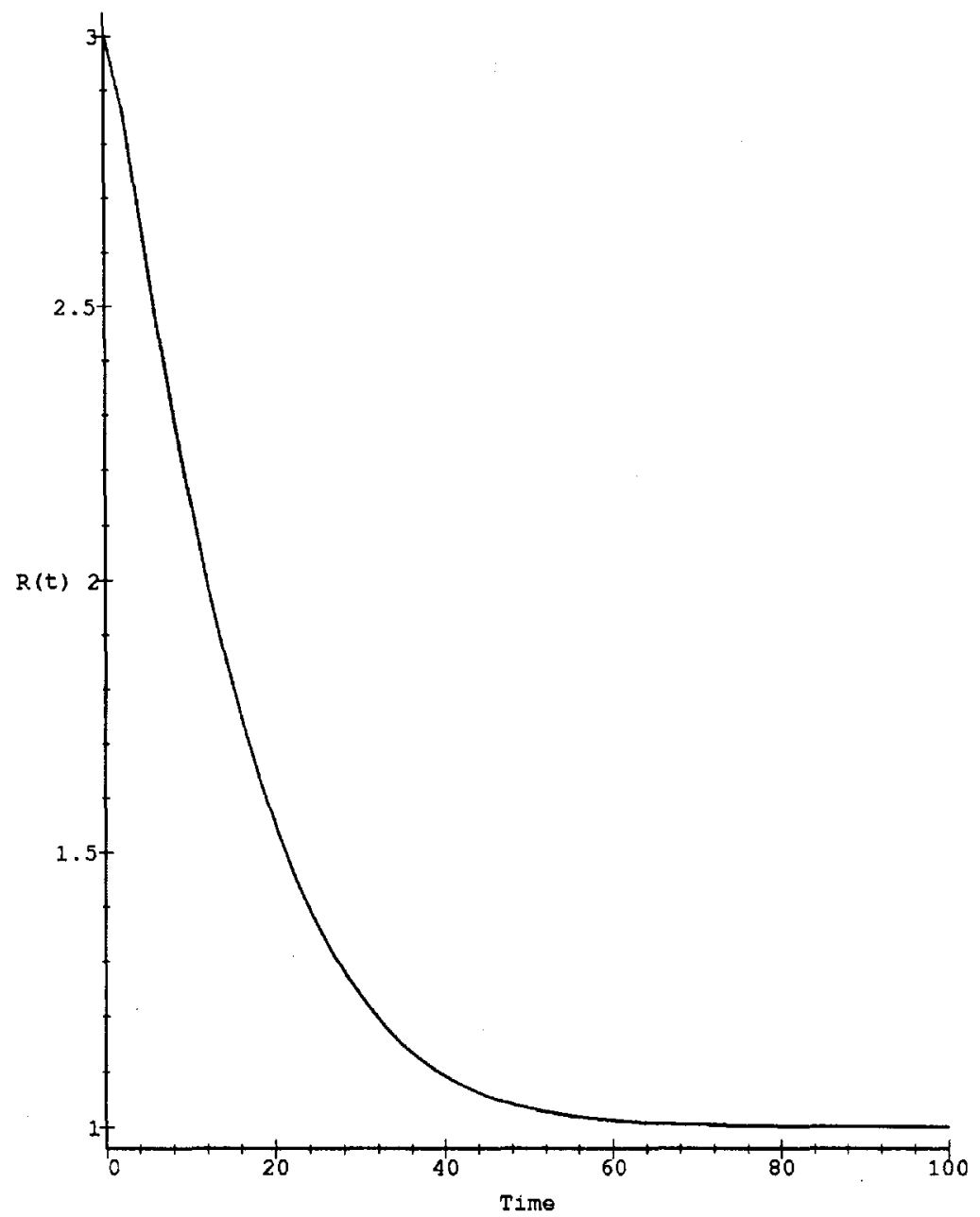

FIGURE 7 Tumour regression using enhanced apoptosis. In all plots $r_{\mathrm{m}}=1, \eta_{0}=0.5, \eta_{1}=1.1, R_{0}=1.0, \gamma=0.2, T=1$.

\section{CONCLUSION}

Following closely previous experimental work (Holmgren et al., 1995, Folkman, 1995, O'Reilly, et al. 1994, 1996) we have presented a quantitative model of the growth and regression of micrometastases. We have established the relevant parameters in the model which may, in principle, be identified by experiment so that the model can be easily run using experimentally determined or available parameter values (see for example Holmgren et al. (1995) for measures of proliferation and apoptotic indices).

By assuming a local imbalance between proliferation and apoptosis, the model shows how a micrometastasis can grow and reach a steady state even in absence of necrosis. The model predicts that, even in a dormant state, at any radius there will be a radial migration of cancer cells, and we note that flows of tumour cells away from central capillary have indeed been reported in tumour cords in experimental animals, e.g. Moore et al. (1984, 1985): The model can follow the development of a micrometastasis provided that its radius never becomes so large that the nutrient concentration in the outer regions drops to such a value that necrosis sets in; and on this point we note that in the experiments of Holmgren et al. (1995) there was no sign of necrosis in the micrometastoses. The model could be extended 
to cover the effects of angiogenesis in much the same way as similar effects have been modelled in the case of primary tumours (Chaplain and Sleeman, 1990, Chaplain et al., 1995; Byrne and Chaplain 1995b, 1996).

The model can be extended in a number of other ways as well. For instance, one could consider the situation were the micrometastasis radius $R(t)$ becomes so large that the oxygen concentration drops to the point where in outer regions cells no longer become viable and necrosis sets in. This situation is relevant to the tumour cords found in a number of human and animal tumours, which are cylindrical cuffs that separate central blood vessels from areas of gross necrosis, e.g. Moore et al. (1984, 1985). Another way in which the model could be extended is to consider a apoptotic index which is a function of radial distance away from the central capillary.

As indicated in the previous section, it is possible using the model to investigate the effects of increasing the rate of apoptosis, which causes the micrometastasis to shrink in radius, i.e. regress. It may thus be of some use in the proposed clinical technique of dormancy therapy in which an angiogenesis inhibitor is used to cause a micrometastasis to regress. It is also straightforward to investigate the effect on growth of a decrease in the rate of apoptosis, caused by the removal of the primary tumour for instance.

\section{Acknowledgements}

The authors would like to thank Dr Brian Dixon of the Research School of Medicine at the University of Leeds for useful discussions.

\section{References}

[1] Adam, J. A. (1991). Diffusion models of prevascular and vascular tumour growth, in Mathematical Population Dynamics, Lecture Notes in Pure and Applied Mathematics, Marcel Dekker, New York. pp. 625-652.

[2] Adam, J. A. and Noren, R. D. (1993). Equilibrium model of a vascularized spherical carcinoma with central necrosis - some properties of the solution, J. Math. Biol., 31, $735-745$.
[3] Bellamy, C. O. C., Malcolmson, R. D. G., Harrison, D. J. and Wyllie, A. H. (1995). Cell death in health and disease: the biology and regulation of apoptosis, Semin Cancer Biol., 6, 3-16.

[4] Byrne, H. M. and Chaplain, M. A. J. (1995a). The growth of nonnecrotic tumours in the presence and absence of inhibitors, Math. Biosci, 130, 151-181.

[5] Byrne, H. M. and Chaplain, M. A. (1995b). Mathematical models for tumour angiogenesis: numerical solutions and nonlinear wave solutions, Bull. Math. Biol., 57(3), 461-486.

[6] Byrne, H. M. and Chaplain, M. A. J. (1996). The growth of necrotic tumours in the presence and absence of inhibitors, Math. Bio. Sci., 135, 187-216.

[7] Burton, A. C. (1966). Rate of growth of solid tumours on a problem of diffusion, Growth, 30, 157-176.

[8] Chaplain, M. A. and Sleeman, B. D. (1990). A mathematical model for the production and secretion of tumour angiogenesis factor in tumours, IMA J. Math. Appl. Med. Biol., 7, $93-108$.

[9] Chaplain, M. A. and Sleeman, B. D. (1993). Modelling the growth of solid tumours and incorporating a method for their classification using nonlinear elasticity theory, J. Math. Biol., 31, 431-473.

[10] Chaplain, M. A., Giles, S. M., Sleeman, B. D. and Jarvis, R. J. (1995). A Mathematical analysis of a model for tumour angiogenesis, J. Math. Biol., 33, 744-770.

[11] Chaplain, M. A. (1996). A vascular Growth, angiogenesis and vascular growth in solid tumours: The mathematical modelling of the stages of tumour development, Math. Comput. Modelling, 23(6), 47-87.

[12] Deakin, A. S. (1975). Model for the growth on an in vitro tumour, Growth, 39, 155-165.

[13] Folkman, J. (1976). The vascularization tumours, Sci. Am., 234, $58-73$.

[14] Folkman, J. (1985). Tumor angiogenesis, Adv. Can. Res., $43,175-203$.

[15] Folkman, J. (1995). Angiogenesis in cancer, vascular, rheumatoid and other disease, Nature Medicine, 1(2), $27-31$

[16] Greenspan, H. P. (1972). Models for the growth of a solid tumour by diffusion, Stud. Appl. Math., 52, 317-340.

[17] Greenspan, H. P. (1976). On the growth and stability of cell cultures and solid tumours, J. Theo. Biol., 56, 229-242.

[18] Holmgren, L., O'Reilly, M. S. and Folkman, J. (1995). Dormancy of micrometastases: Balanced proliferation and apoptosis in the presence of angiogenesis suppression. Nature Medicine, 1(2), 149-153.

[19] McElwain, D. L. S. and Ponzo, P. J. (1977). A model for the growth of a solid tumour with non-uniform oxygen consumption, Math. Biosci., 35, 267-279.

[20] McElwain, D. L. S. and Morris, L. E. (1978). Apoptosis as a volume loss mechanism in mathematical models of solid tumour growth, Math. Biosci, 39, 147-157.

[21] McElwain, D. L. S. and Pettet, G. J. (1993). Cell migration in multicell spheroids: swimming against the tide, Bull. Math. Biol., 55(3), 655-674

[22] Moore, J. V., Hopkins, H. A. and Looney, W. B. (1984) Tumour-cord parameters in two rat hepatomas that differ in their radiobiological oxygenation status. Radiat. Environ Biophy., 23, 213-222.

[23] Moore, J. V., Haselton, P. S. and Buckley, C. H. (1985). Tumour cords in 52 human bronchial and cervical squamous cell carcinomas: Inferences for their cellular kinetics and radiobiology. Br. J. Cancer, 51, 407-413.

[24] O'Reilly, M. S., Holmgren, L., Shing, Y., Chen, C., Rosenthal, R. A., Moses, M., Lane, W. S., Chao, Y., Sage, E. H. 
and Folkman, J. (1994). Angiostatin: A novel angiogenesis inhibitor that mediates the suppression of metastases by a Lewis lung carcinoma, Cell, 79, 315-328.

[25] O'Reilly, M. S., Holmgren, L., Chen, C. and Folkman, J. (1996). Angiostatin induces and sustains dormancy of human primary tumours in mice, Nature Medicine, 2(6), $689-692$

[26] Sleeman, B. D. (1996). Solid tumour growth: a case study in mathematical biology, in Nonlinear Mathematics and its
Applications, ed. Philip, J. A., (Cambridge University Press, Cambridge). 237-256.

[27] Steller, H. (1995). Mechanisms and genes of cellular suicide, Science, 267, 1445-1456.

[28] Thompson, C. B. (1995). Apoptosis in the pathogenesis and treatment of disease, Science, 267, 1456-1462.

[29] Woodruff, M. (1980). The Interactions of Cancer and the Host, (Grune and Stratton, New York).

[30] Woodruff, M. (1990). Cellular Variation and Adaptation in Cancer, (Oxford University Press, New York). 


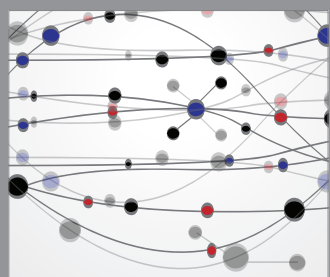

The Scientific World Journal
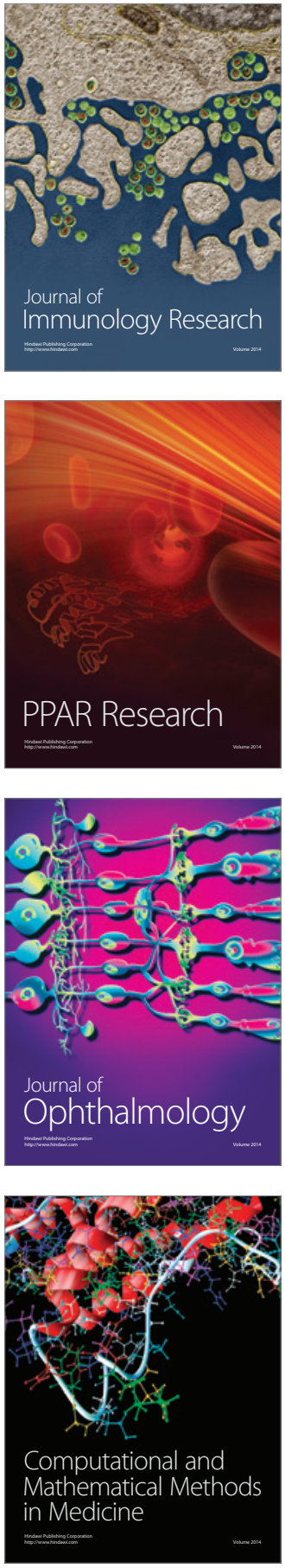

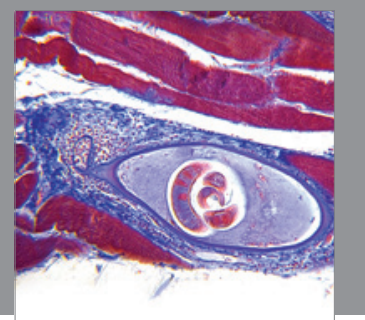

Gastroenterology

Research and Practice
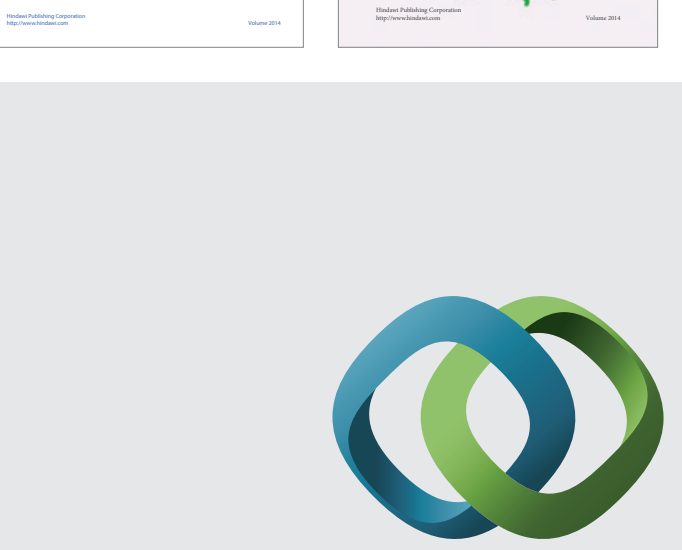

\section{Hindawi}

Submit your manuscripts at

http://www.hindawi.com
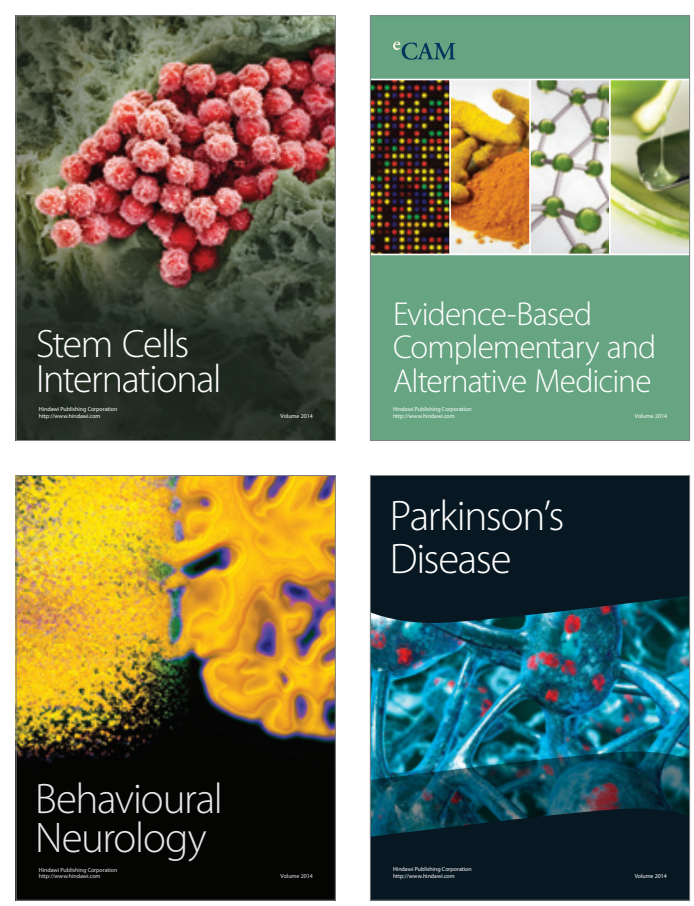

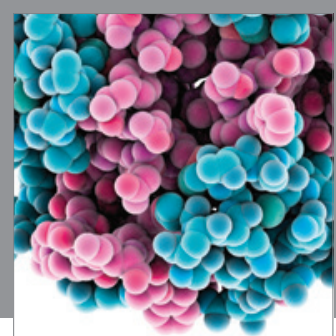

Journal of
Diabetes Research

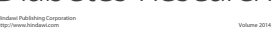

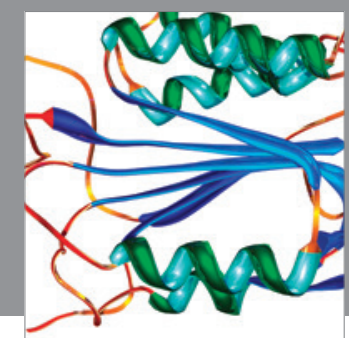

Disease Markers
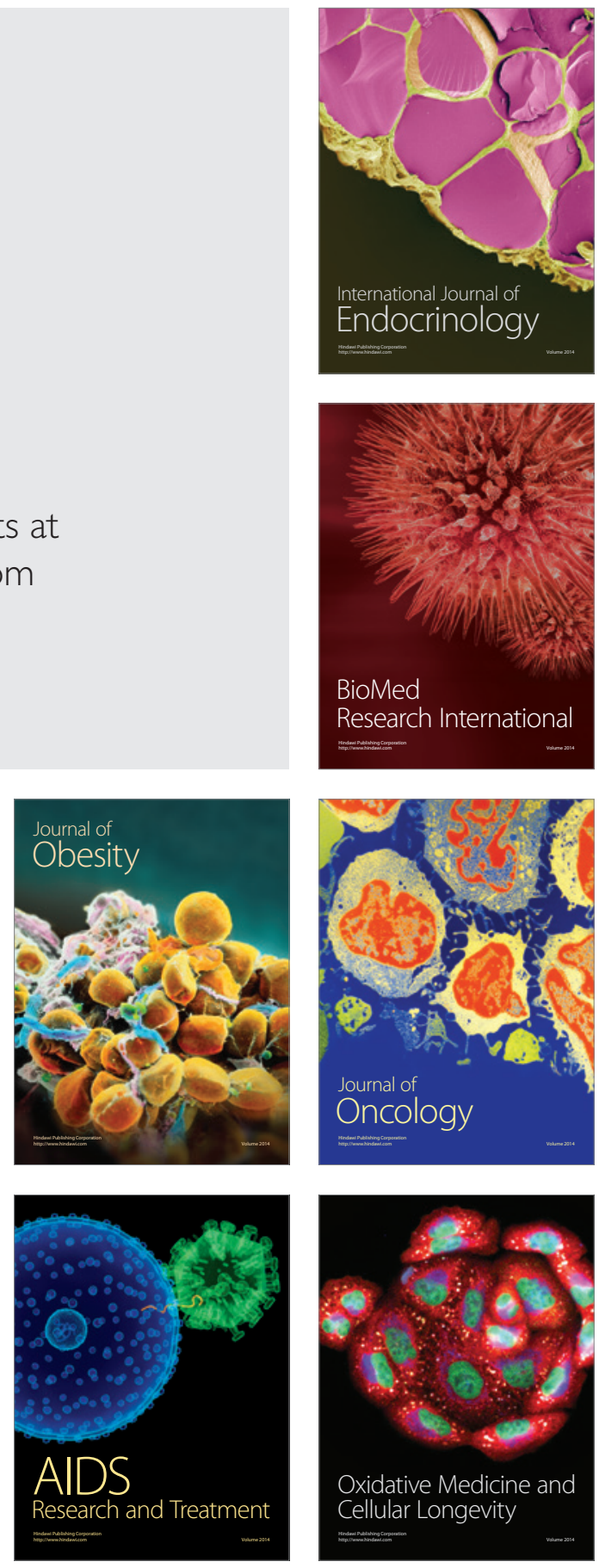